\title{
What is the clinical impact of the endometrial receptivity array in PGT-A and oocyte donation cycles?
}

\author{
Ana Raquel Neves ${ }^{1}$ (1) $\cdot$ Marta Devesa ${ }^{2} \cdot$ Francisca Martínez $^{2} \cdot$ Sandra Garcia-Martinez $^{2} \cdot$ Ignacio Rodriguez $^{2}$. \\ Nikolaos P. Polyzos ${ }^{2,3,4} \cdot$ Buenaventura Coroleu $^{2}$
}

Received: 28 May 2019 / Accepted: 16 July 2019 / Published online: 27 July 2019

(C) Springer Science+Business Media, LLC, part of Springer Nature 2019

\begin{abstract}
Purpose To evaluate the influence of the endometrial receptivity array (ERA) test on the implantation rate (IR) and pregnancy rate (PR) in patients with previous failed euploid embryo transfers (Euploid-ET) or oocyte donation embryo transfers (Donor-ET).

Methods Single-center retrospective study of patients with $\geq 1$ previous failed Euploi-ET $(n=24)$ or $\geq 2$ failed Donor-ET $(n=32)$ who underwent an ERA test and a post-ERA Euploid-ET/Donor-ET between 2012 and 2018. Controls were patients with $\geq 1$ previously failed Euploid-ET $(n=119)$ or $\geq 2$ failed Donor-ET ( $n=158)$ who underwent Euploid-ET/Donor-ET during the same period without performing an ERA test. Only blastocyst stage embryos were included. IR/PR was compared between the postERA ET and the last ET in the control group.

Results There was no statistically significant difference regarding IR [55.6\% (34.6-76.5\%) vs. $65.0 \%$ (56.9-73.1\%)] nor PR $(58.3 \%$ vs. $70.6 \%, p=0.238)$ in the Euploid-ET ERA vs. Euploid-ET control groups. In the Donor-ET arm, both IR [26.8\% (12.3$41.4 \%)$ vs. $57.2 \%(50.1-64.3 \%)]$ and PR (34.4\% vs. $65.2 \%, p=0.001)$ were significantly lower in the ERA group. Multivariate analysis confirmed that performing an ERA test did not influence the PR in the Euploid-ET arm and was associated with a diminished PR in the Donor-ET arm. In the ERA group, $41.1 \%$ patients were non-receptive (NR). No significant difference was found regarding IR/PR in NR vs. receptive patients in both Euploid-ET/Donor-ET arms.

Conclusions In our sample, the performance of an ERA test did not improve pregnancy outcomes. Future prospective studies in larger samples are needed to confirm the role of the ERA test in Euploid-ET/Donor-ET.
\end{abstract}

Keywords Endometrial receptivity array $\cdot$ Implantation failure $\cdot$ Oocyte donation $\cdot$ PGT-A

\section{Background}

After ovulation, endometrial development culminates with a defined period of maximal endometrial receptivity. This

Ana Raquel Neves

anaraquel.lneves@gmail.com

Marta Devesa

mardev@dexeus.com

Francisca Martínez

pacmar@dexeus.com

Sandra Garcia-Martinez

garsan@dexeus.com

Ignacio Rodriguez

nacrod@dexeus.com

Nikolaos P. Polyzos

nikpol@dexeus.com window of implantation takes place in the mid-secretory phase, from postovulatory day 6 to 10. During this period, tangled molecular interactions mediate the histological alterations that enable the complex process of apposition,

Buenaventura Coroleu

vencor@dexeus.com

1 Department of Obstetrics and Gynecology, Coimbra Hospital and University Centre, Rua Augusta 17, 3000-045 Coimbra, Portugal

2 Department of Obstetrics, Gynecology and Reproductive Medicine, Dexeus University Hospital, 08028 Barcelona, Spain

3 Faculty of Medicine and Pharmacy, Department of Surgical and Clinical Science, Vrije Universiteit Brussel, 1050 Brussels, Belgium

4 Department of Clinical Medicine, Faculty of Health, Aarhus University, 8000 Aarhus, Denmark 
adhesion, and invasion of the trophoblast between the endometrial epithelial cells [1].

Although assisted reproduction techniques have managed to overcome many barriers in the fertility process, implantation still remains a challenge for both physicians and embryologists. Despite the extensive investigation in the field of implantation failure (IF), we are still lacking reliable information on the evaluation of the endometrial factor [2]. A number of markers have been studied as potential biomarkers of receptivity, including ultrasound, histological, and molecular markers. However, none of them has proven their ability to predict clinical pregnancy [2].

Recently, investigation of the endometrial gene expression profile has gained ground and development of endometrial gene expression arrays, such as the Endometrial Receptivity Analysis (ERA) test, have been considered the new step in the exploration of endometrial receptivity.

The ERA test is a microarray-based machine-learning predictive model that classifies the endometrium according to its receptivity status, providing recommendations on the endometrial preparation before embryo transfer (ET) [3]. This test has been studied both in patients with and without previous IF yielding conflicting results [4-7].

One important limitation of these studies is that they do not account for the contribution of aneuploidy to IF. Patients with previous failed in vitro fertilization (IVF) cycles have been shown to have higher rates of aneuploid embryos when compared to fertile couples [8]. Furthermore, taking into account that IF increases with maternal age and more than $60 \%$ of embryos in women over 38 years are aneuploid, adjusting for this confounder seems to be of utmost importance when analyzing the effect of the endometrial factor on IF [9-11]. Another recognized strategy to limit the influence of maternal age in implantation is oocyte donation. A $37-40 \%$ implantation rate (IR) has been reported in oocyte donation cycles, similar to the mean IR in IVF cycles [12-15].

We hypothesize that controlling for the embryonic factor will allow for a more representative assessment of the endometrial factor.

The aim of this study was to evaluate the influence of the ERA test in the reproductive outcomes of a cohort of patients with previous IF in IVF coupled with preimplantation testing for embryonic aneuploidy (PGT-A) cycles or oocyte donation cycles.

\section{Methods}

We conducted a single-center retrospective study, in a university-affiliated private fertility clinic, including patients with previous IF who underwent an ERA test until March 2018 and a post-ERA ET between October 2012 and December 2018. Controls were patients with prior IF, with the same selection criteria, who underwent a subsequent ET without a previous ERA test during the study period (index cycle).

\section{Implantation failure definition}

In the context of this study IF was defined as:

$-\geq 1$ previous ET failure after the transfer of an euploid embryo, regardless of previous transfers of embryos of unknown ploidy status

$-\geq 2$ previous ET failure from reception cycles

Cut offs for the number of failed cycles were selected considering a probability of IF of $30 \%$; half of the probability of IF described for blastocysts in the general IVF population [12]. Taking into account the $40 \%$ IR for blastocyst transfer, the likelihood of two blastocysts failing to implant is $36 \%$, very similar to the likelihood of IF for 1 euploid embryo $(30 \%)[12,16]$.

\section{Patients' selection criteria}

The study group included patients who had undergone one of the following: (a) $\geq 1$ previous failed euploid embryo transfer (Euploid-ET) and (b) $\geq 2$ previous failed ET from reception cycles (Donor-ET) without PGT-A [17]. Patients were included when failed cycles were followed by an ERA test and a post-ERA ET under the same cycle type (Euploid-ET ERA/ Donor-ET ERA) taking into account the ERA recommendation. The control group comprised patients with the same inclusion criteria, whose failed cycles were followed by an ET with the same protocol (Euploid-ET control or Donor-ET control) without a previous ERA test.

All patients included underwent the following workup: two and three dimensional vaginal ultrasound (with hysteroscopy or hysterosonography when indicated), karyotypes of both partners, thyroid stimulating hormone (TSH), lupus anticoagulant, anticardiolipin antibodies $\operatorname{IgG}$ and $\mathrm{IgM}$, antithrombin III, protein $\mathrm{C}$, protein $\mathrm{S}$, serum homocystine, prothrombin G20210A mutation, and factor V Leiden mutation. Patients were included when normal results were obtained in the workup.

Exclusion criteria were cleavage stage embryo transfers, nonoperated hydrosalpinx, untreated congenital uterine anomaly, untreated submucous myomas or endometrial polyps, difficult embryo transfers (those with a difficult insertion of a malleable Wallace ${ }^{\circledR}$ stylet (Simcare Ltd., Colchester, Sussex, UK) or requiring additional instrumentation), untreated inherited or acquired thrombophilia, and recurrent pregnancy loss ( $\geq 3$ previous miscarriages). As per our institutional protocol, endometrial thickness is measured on the day before ET. Patients with an atrophic endometrium $(<5.5 \mathrm{~mm})$ measured on the day before ET were excluded in order to eliminate a potential bias in the interpretation of implantation failure. 
Cycles that did not achieve ET were also excluded from the analysis.

The selection diagrams for the ERA and control groups are displayed in Fig. 1.

\section{Ovarian stimulation and oocyte retrieval}

All IVF or intracytoplasmic sperm injection (ICSI) cycles were performed under controlled ovarian stimulation with gonadotropins and pituitary suppression with GnRH analogs (agonists or antagonists) according to previously described protocols [18]. When at least three follicles $>18 \mathrm{~mm}$ in diameter were observed, ovulation was triggered with $250-\mu \mathrm{g}$ recombinant HCG (Ovitrelle; Merck Serono, Madrid, Spain) or $0.2 \mathrm{mg}$ triptoreline (Decapeptyl; Ipsen Farma, Barcelona, Spain). According to our institutional protocol, GnRH agonist trigger is applied in patients at risk of ovarian hyperresponse syndrome. In the study population, no patient presented hyperresponse; therefore, GnRH agonist trigger was not routinely performed. Transvaginal oocyte retrieval was performed $36 \mathrm{~h}$ later under sedation with propofol. In all cases of fresh ET, ovulation was triggered with recombinant HCG.

In oocyte reception cycles, donor selection and cycle protocol were as previously described by Clua et al [14].

\section{Embryo manipulation and PGT-A}

Gamete manipulation, oocyte insemination, and embryo culture were performed according to standard protocols used in the IVF laboratory of the study's center [19].
Embryo quality was assessed according to number and regularity of blastomeres, percentage of fragmentation, and multinucleation. In accordance with our embryo scoring system, embryos graded with a score $\geq 8$ on a scale of 1 to 10 were considered good-quality embryos until December 2015 [14]. From then onwards, good-quality embryos were classified as A or B in a scale from D to A as described by the ASEBIR consensus on embryo assessment [20].

PGT-A was performed in cleavage stage embryos until December 2014, and trophoectoderm biopsy was performed from then onwards. Both protocols have been previously described by Coll et al [19].

\section{Embryo transfer}

Only blastocyst stage transfers were included in the study.

Regarding fresh ET cycles, embryos were transferred on day 5 of the cycle. The luteal phase was supported with $200 \mathrm{mg}$ of micronized vaginal progesterone t.i.d.

All frozen ET were performed with surplus embryos frozen after oocyte retrieval with the vitrification method, according to previously established standard protocols [21]. Endometrial priming was performed as previously described by Martínez et al [13]. Briefly, patients underwent treatment with $2 \mathrm{mg}$ estradiol valerate t.i.d for 12-14 days and then vaginal micronized progesterone was added at $200 \mathrm{mg}$ t.i.d. ET was performed on day $\mathrm{P}+5$ in the control group and according to the ERA test result in the study group.

In all cases, ET was performed under ultrasound guidance [22], and luteal phase support was maintained for 14 days, until a serum $\beta$-hCG determination and, in case of a positive

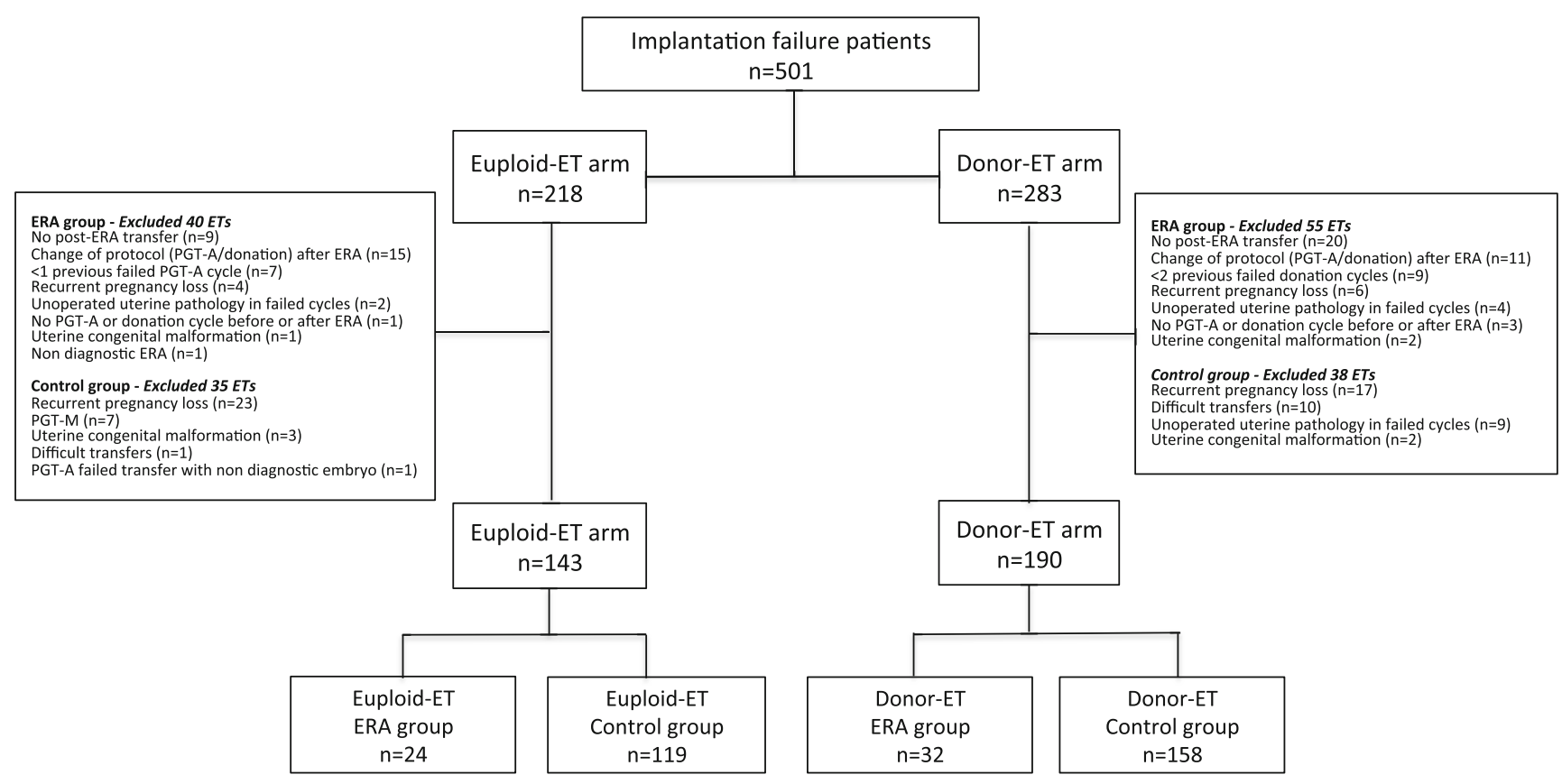

Fig. 1 Selection diagram. PGT-M, preimplantation genetic testing for monogenic defects 
result, until confirmation of the pregnancy by the presence of fetal heartbeat on an ultrasound scan.

\section{Endometrial biopsy}

In all patients included in the study group, the ERA test was performed in a hormone replacement therapy (HRT) mock cycle. Patients underwent treatment with $2 \mathrm{mg}$ E2 valerate t.i.d for 12-14 days and then $200 \mathrm{mg}$ of micronized vaginal Progesterone t.i.d was added on days $13-15$ by night $(\mathrm{P}+0)$. Endometrial biopsy was performed on day $\mathrm{P}+5$ using a Pipelle ${ }^{\circledR}$ endometrial sampler (Laboratoire CCD, Paris, France) or similar device, under sterile conditions. The ERA test was performed as described by Ruiz-Alonso et al [6]. ERA results were classified as receptive, pre-receptive, or post-receptive, and recommendations regarding endometrial preparation were considered in the following cycle.

\section{Pregnancy outcome measures}

The IR was calculated by dividing the number of intrauterine gestational sacs observed on ultrasound by the number of embryos transferred. Clinical pregnancy (CP) was defined by ultrasonographic visualization of one or more intrauterine gestational sacs containing at least one embryo with heartbeat.

\section{Statistical analysis}

Continuous variables were expressed as mean and standard deviation, rates as percentages, and $95 \%$ confidence intervals (CI) and categorical variables as frequencies and percentages. Univariate analysis was carried out to describe and compare the baseline and characteristics between patients in the ERA and control groups and to explore correlations between variables. Univariate analysis was also performed to compare pregnancy outcome measures between the first post-ERA transfer, in the study group, and the index cycle in the control group. Categorical variables were compared using the chisquare test or the Fisher exact test according to the Cochrane rules. Continuous variables were compared using the Student's $T$ test. All tests were two-tailed, and a $p<0.05$ was considered statistically significant. A multinomial logistic regression (LR) model was also constructed to analyze possible predictors of CP in both Euploid-ET and Donor-ET arms.

We further performed propensity score analysis in order to match characteristics between the ERA and control groups based on factors that may affect the $\mathrm{CP}$ rate. The propensity score is the estimated probability that a given patient would have been assigned to the ERA test, given a particular set of variables. In this case, patients were matched for age, number of previous failed ET, and number of previously transferred embryos. We then performed a logistic regression using $\mathrm{CP}$ as the dependent variable and the treatment group (ERA/control) and the four quartiles of the propensity score for performing ERA as predictors $(\mathrm{Q} 1, \leq 6.3 \%$; Q2, 6.4-11.7\%; Q3, 11.8$18.2 \%$; Q4, $\geq 18.3 \%$ )

Statistical analyses were performed with IBM@ SPSS $\odot$ Statistics v 22 and SAS Institute Inc. 2014. Base SAS ${ }^{\circledR} 9.4$ Procedures Guide. Cary NC: SAS Institute Inc.

\section{Results}

A total of 333 patients with IF were included in the study. The proportion of ERA tests performed was the same in the Euploid-ET group, $16.8 \%$ (24/143), and in the Donor-ET group, $16.8 \%$ (32/190) (Fig. 1).

There were a total of 212 clinical pregnancies, with a total clinical pregnancy rate (CPR) of $68.5 \%$ (98/143) in the EuploidET group and 60.0\% (114/190) in the Donor-ET group.

\section{Euploid-ET arm}

When comparing the Euploid-ET ERA group with the Euploid-ET control group, patients in the Euploid-ET ERA group had a higher total number of previously failed cycles $(2.29 \pm 1.43$ vs. $1.59 \pm 1.25, p=0.015)$, a higher number of failed PGT-A cycles $(1.54 \pm 0.72$ vs. $1.14 \pm 0.40, p=0.014)$, and a higher number of previously transferred euploid embryos $(1.75 \pm 0.85$ vs. $1.33 \pm 0.55, p=0.027)$. The IR [55.6\% (34.6-76.5\%) vs. $65.0 \%(56.9-73.1 \%)]$ and CPR (58.3\% vs. $70.6 \%, p=0.238$ ) were lower in the Euploid-ET ERA group, although this difference was not statistically significant (Table 1). Multivariate LR and propensity score analysis showed that none of the analyzed variables was a significant predictor of the CPR (Tables 3a and 4a, respectively).

\section{Donor-ET arm}

When comparing the Donor-ET ERA group with the DonorET control group, patients in the Donor-ET ERA group also had a higher number of previously failed Donor-ET cycles $(2.87 \pm 0.96$ vs. $2.49 \pm 0.89, p=0.035)$. The mean number of embryos transferred in the index cycle was lower in the Donor-ET ERA group $(1.16 \pm 0.57$ vs $1.37 \pm 0.54, p=$ $0.040)$. Both IR $[26.8 \%(12.3-41.4 \%)$ vs. $57.2 \%(50.1-$ $64.3 \%)$ ] and CPR (34.4\% vs. $65.2 \%, p=0.001)$ were also significantly lower in the Donor-ET ERA group when compared with the Donor-ET control group (Table 2). Multivariate LR revealed that transferring top quality embryos (TQE) and not performing the ERA test were significantly associated with CP [respectively, adjOR 3.93, 95\% (2.03-7.57) and adjOR 3.60, 95\% CI (1.46-8.86)] (Table 3b). Propensity score analysis confirmed that not performing ERA significantly increased the CPR [AdjOR 3.76 (95\% CI 1.65-8.60)] (Table 4b). 
Table 1 Patients' baseline characteristics and pregnancy outcomes in the Euploid-ET arm

\begin{tabular}{|c|c|c|c|}
\hline & $\begin{array}{l}\text { Euploid-ET } \\
\text { ERA } \\
(n=24)\end{array}$ & $\begin{array}{l}\text { Euploid-ET } \\
\text { Control } \\
(n=119)\end{array}$ & $p$ value \\
\hline \multicolumn{4}{|l|}{ Demographic data } \\
\hline Age & $39.25 \pm 3.99$ & $39.18 \pm 3.80$ & 0.932 \\
\hline BMI $\left(\mathrm{kg} / \mathrm{m}^{2}\right)$ & $23.21 \pm 4.10$ & $22.67 \pm 3.52$ & 0.570 \\
\hline \multicolumn{4}{|l|}{ Previous embryo transfers } \\
\hline Number of previously failed Euploid-ET & $1.54 \pm 0.72$ & $1.14 \pm 0.40$ & 0.014 \\
\hline $\begin{array}{l}\text { Number of previously transferred euploid } \\
\text { embryos } \\
\text { Index cycle }\end{array}$ & $1.75 \pm 0.85$ & $1.33 \pm 0.55$ & 0.027 \\
\hline \multicolumn{4}{|l|}{ Type of transfer } \\
\hline $\begin{array}{l}\text { Fresh } \\
\text { Frozen }\end{array}$ & $\begin{array}{l}0 \\
100 \%(24)\end{array}$ & $\begin{array}{l}2.5 \%(3) \\
97.5 \%(116)\end{array}$ & 0.432 \\
\hline Number of euploid embryos transferred & $1.13 \pm 0.34$ & $1.18 \pm 0.38$ & 0.542 \\
\hline Top quality embryos & $54.2 \%(13)$ & $49.6 \%(59)$ & 0.682 \\
\hline Progesterone on the day before transfer $(\mathrm{ng} / \mathrm{ml})$ & $10.64 \pm 4.73$ & $12.23 \pm 5.42$ & 0.201 \\
\hline Estradiol on the day before transfer $(\mathrm{pg} / \mathrm{ml})$ & $229.84 \pm 85.48$ & $195.15 \pm 90.49$ & 0.100 \\
\hline $\begin{array}{l}\text { Endometrial thickness on the day before } \\
\text { transfer }(\mathrm{mm})\end{array}$ & $9.91 \pm 1.82$ & $10.43 \pm 1.94$ & 0.245 \\
\hline Implantation rate & $55.6 \%(34.6-76.5 \%)$ & $65.0 \%(56.9-73.1 \%)$ & \\
\hline Clinical pregnancy rate & $58.3 \%(14)$ & $70.6 \%(84)$ & 0.238 \\
\hline
\end{tabular}

Quantitative variables are presented as mean $\pm \mathrm{SD}$ and qualitative variables as \% (n)

\begin{tabular}{llll}
\hline & $\begin{array}{l}\text { Donor-ET } \\
\text { ERA } \\
(n=32)\end{array}$ & $\begin{array}{l}\text { Donor-ET } \\
\text { Control } \\
(n=158)\end{array}$ & $p$ value \\
& & & \\
\hline Demographic data & $42.19 \pm 3.34$ & $43.40 \pm 4.13$ & 0.121 \\
Age & $23.70 \pm 3.70$ & $23.86 \pm 4.30$ & 0.859 \\
BMI (kg/m ${ }^{2}$ ) & & & \\
Previous embryo transfers & $2.87 \pm 0.96$ & $2.49 \pm 0.89$ & 0.035 \\
Number of previously failed Donor-ET & $3.97 \pm 1.66$ & $3.66 \pm 1.68$ & 0.350 \\
Number of previously transferred reception & & & \\
$\quad$ embryos & & & 0.853 \\
Index cycle & & $26.6 \%(42)$ & \\
Type of transfer & $25 \%(8)$ & $73.4 \%(116)$ & $1.37 \pm 0.54$ \\
Fresh & $75 \%(24)$ & $55.7 \%(88)$ & 0.040 \\
Frozen & $1.16 \pm 0.57$ & $12.35 \pm 5.15$ & 0.954 \\
Number of embryos transferred & $56.3 \%(18)$ & $251.31 \pm 209.96$ & 0.220 \\
Top quality embryos & $14.11 \pm 6.39$ & $10.13 \pm 1.86$ & 0.958 \\
Progesterone on the day before transfer $(\mathrm{ng} / \mathrm{mL})$ & & \\
Estradiol on the day before transfer $(\mathrm{pg} / \mathrm{mL})$ & $207.32 \pm 75.67$ & $57.2 \%(50.1-64.3 \%)$ & \\
Endometrial thickness on the day before & $10.11 \pm 1.95$ & $65.2 \%(103)$ & 0.001 \\
$\quad$ transfer (mm) & $26.8 \%(12.3-41.4 \%)$ & \\
Implantation rate & $34.4 \%(11)$ & & \\
Clinical pregnancy rate & & & \\
\hline
\end{tabular}

Quantitative variables are presented as mean $\pm \mathrm{SD}$ and qualitative variables as \% (n) 
Table 3 Multivariate logistic regression using clinical pregnancy as the dependent variable in the Euploid-ET arm (a) and Donor-ET arm (b)

AdjOR (95\% CI)

a)
Age

Group (control/ERA)

Number of previous Euploid-ET

Number of previously transferred euploid embryos

Top quality embryos

b)

Age

Group (control/ERA)

Number of previous Donor-ET

Number of previously transferred reception cycles embryos

Top quality embryos

Number of embryos transferred in the index cycle
$0.93(0.84-1.02)$

$1.55(0.58-4.13)$

$0.65(0.20-2.07)$

$1.09(0.44-2.68)$

$1.71(0.81-3.58)$

$0.97(0.89-1.05)$

$3.60(1.46-8.86)$

$0.91(0.50-1.66)$

$1.06(0.76-1.49)$

$3.93(2.03-7.57)$

$1.88(0.89-3.56)$

\section{ERA subanalysis}

Overall, 58.9\% (33/56) patients presented a receptive (R) endometrium, and $41.1 \%$ (23/56) were nonreceptive (NR). Among NR patients, $69.6 \%(16 / 23)$ were pre-receptive, $17.4 \%(4 / 23)$ were post-receptive, $8.7 \%$ (2/23) were late receptive, and $4.3 \%(1 / 23)$ were early receptive. The prevalence of NR endometrium was $33.3 \%(8 / 24)$ in the Euploid-ET ERA group and $46.9 \%(15 / 32)$ in the Donor-ET ERA. We found no statistically significant difference regarding IR and CPR when comparing NR and R patients in both Euploid-ET ERA [respectively, $40.0 \%(2.2-78 \%)$ vs. $64.7 \%$ (38.4$91.0 \%)$ and $50.0 \%$ vs. $62.5 \%, p=0.673]$ and Donor-ET ERA groups [respectively, $20.0 \%(0.1-39.9 \%)$ vs. $33.3 \%$ $(10.3-56.3 \%)$ and $26.7 \%$ vs. $41.2 \%, p=0.388]$.

Table 4 Propensity score analysis using clinical pregnancy as the dependent variable in the Euploid-ET arm (a) and Donor-ET arm (b). P (ERA) Q4 was used as the reference group. In the Euploid-ET arm, there was no patient in the P (ERA) Q1 subgroup. In the Donor-ET arm, there was only one patient in the P (ERA) Q1 subgroup

AdjOR (95\% CI)

a)

ERA (non-ERA/ERA)

$1.94(0.75-5.00)$

P (ERA) Q2

$0.69(0.25-1.89)$

$P$ (ERA) Q3

$0.66(0.26-1.70)$

b)

ERA (non-ERA/ERA)

$3.76(1.65-8.60)$

P (ERA) Q1

$\mathrm{P}$ (ERA) Q2

$P$ (ERA) Q3

$0.00(0.00-0.00)$

$0.68(0.32-1.48)$

$0.94(0.44-1.99)$
Finally, taking into account the recent controversies regarding the effect of the endometrial injury on CPR [23], we analyzed the IR and CPR according to the interval from biopsy to ET. Only two patients in the Euploid-ET ERA group and none in the Donor-ET ERA group performed the ET in the first month after the biopsy. In the Euploid-ET ERA group, IR and CPR were higher in the second month after the biopsy (76.9\% and $81.8 \%$, respectively), while in the Donor-ET ERA group, both IR and CPR were higher in the third month after the biopsy ( $37.5 \%$ and $50 \%$, respectively).

\section{Discussion}

In the present study, the ERA test did not improve pregnancy outcomes neither in patients with at least one failed EuploidET nor in patients with at least two failed Donor-ETs.

To our knowledge, this is the first study to correlate the ERA test with pregnancy outcomes in both PGT-A cycles and oocyte donation cycles including a control population.

Previous studies have reported the clinical impact of the ERA test both in RIF and non-RIF populations yielding conflicting results [4-7]. However, none of these studies accounted for the embryo aneuploidy rate, which may have influenced the reported findings. More recently, Tan et al reported the clinical utility of the ERA test in Euploid-ET, with similar implantation rates, ongoing pregnancy rates, and live birth rates in R and NR patients [24]. However, in this study, the authors did not include a control group without a previous ERA test. Bassil et al was the first to compare pregnancy outcomes in a population performing ERA and a control population and found no improvement in the ongoing pregnancy rate [5]. Nevertheless, in this study, the authors did not account for the embryo ploidy status.

Regarding the Euploid-ET arm, our data show that, after adjusting for potential confounders, no statistically significant difference was found regarding the clinical pregnancy rates in patients who had performed the ERA test when compared to controls. This finding had not been previously reported. This discrepancy could be related to the lack of improvement of the basal endometrial receptivity by personalized endometrial preparation according to ERA test in the study population.

Multivariate analysis in the Euploid-ET arm also revealed that the transfer of TQE was not associated with the CPR, which is in line with previous studies reporting only mild associations between embryo ploidy and morphology [25, 26].

Regarding the Donor-ET arm, multivariate logistic regression revealed that the CPR was significantly reduced in the ERA group, a finding had not been previously reported. This unexpected finding could also be related to the lack of response of the endometrium to the personalized endometrial preparation of recipients of donated oocytes in our sample. 
An ERA group subanalysis further revealed an overall receptivity rate of $58.9 \%$. While the majority of previous studies reported a receptive endometrium in $72.5-76 \%$ RIF patients and $84.9-88 \%$ non-RIF patients [4, 6, 7], Tan et al reported an overall receptivity rate of $55.7 \%$, and Bassil et al reported a receptivity rate of $35.8 \%$ in patients with up to two previous failed ET [5, 24]. In the Euploid-ET ERA group, the receptivity rate was $66.7 \%$, similar to the results presented by Tan et al $(62.5 \%)$ [24]; while in the Donor-ET ERA group, $56.1 \%$ of patients presented a receptive endometrium, lower than observed by Mahajan et al (68\%) [4]. The majority of NR patients presented a pre-receptive endometrium, which is in line with previous studies [4-7, 24].

We found a lower IR and CPR when comparing NR and R patients in the Euploid-ET ERA arm, although this difference was not statistically significant. Tan et al had previously reported a higher non-significant IR and CPR in NR patients in a population with a mean age $39.25 \pm 3.99$ years, in disagreement with our results [24]. We also found a lower nonsignificant IR and CPR in NR patients in the Donor-ET ERA arm, similar to the figures reported by Mahajan et al [4].

Taking into account the conflicting reports on the effect of the endometrial injury in the reproductive outcomes, we further analyzed both implantation and pregnancy rates according to the interval from biopsy to ET $[23,27]$. Only two ETs in the Euploid-ET ERA group and none in the Donor-ET ERA group were performed in the first month after the biopsy. Therefore, the endometrial injury did not account for our results.

Our results should be interpreted with caution taking into account the small sample size of the ERA groups. Other limitations of our study include its retrospective design and the fact that the groups were not matched for the number of previous ETs. However, multivariate logistic regression analysis revealed that previous ETs were not associated with the pregnancy rate. The fact that both fresh and frozen ET were included could also have influenced our results. Exclusion of fresh cycles was not performed so as not to reduce the sample size. Furthermore, taking into account that the distribution between fresh and frozen ET was similar between both ERA and control groups, we believe our findings were not confounded by this variable. Another limitation was the fact that we did not perform a post-ERA endometrial biopsy to confirm endometrial receptivity after the adjusted endometrial preparation protocol due to patients' financial restrictions.

\section{Conclusion}

In the era of transcriptomics and bioinformatics, the ERA test came as a promising tool providing valuable information on endometrial dating. However, its clinical impact remains controversial. Prospective studies with larger samples are needed to clarify the effect of a personalized embryo transfer in the reproductive outcomes.

Acknowledgments This work was performed under the auspices of the Cátedra de Investigació en Obstetrícia I Ginecologia of the Department of Obstetrics, Gynaecology and Reproduction, Dexeus University Hospital, Universitat Autònoma de Barcelona.

Competing interests The authors declare that they have no competing interests.

Authors' contributions ARN designed the study, performed the literature search, wrote the manuscript, and constructed the tables and figures. MD, FM, NP, and BC contributed to the design of the research study, edited the manuscript, and provided critical review of the manuscript. SGM and IR contributed to data collection and statistical analysis. All authors read and approved the final manuscript.

\section{Compliance with ethical standards}

The study was performed in accordance with the Helsinki Declaration and with approval of the Institutional Review Board. Because the study involved completely de-identified data extraction from electronic medical records, patient consent was not required.

\section{References}

1. Fox C, Morin S, Jeong JW, Scott RT, Lessey BA. Local and systemic factors and implantation: What is the evidence? Fertil Steril. 2016;105(4):873-84.

2. Craciunas L, Gallos I, Chu J, Bourne T, Quenby S, Brosens JJ, et al. Conventional and modern markers of endometrial receptivity: a systematic review and meta-analysis. Hum Reprod Update. 2019;25(2):202-23.

3. Díaz-Gimeno P, Horcajadas JA, Martinez-Conejero JA, Esteban FJ, Alama P, Pellicer A, et al. A genomic diagnostic tool for human endometrial receptivity based on the transcriptomic signature. Fertil Steril. 2011;95(1):50-60.

4. Mahajan N. Endometrial receptivity array: Clinical application. J Hum Reprod Sci. 2015;8(3):121-9.

5. Bassil R, Casper R, Samara N, Hsieh TB, Barzilay E, Orvieto R, et al. Does the endometrial receptivity array really provide personalized embryo transfer? J Assist Reprod Genet. 2018;35(7):1301-5.

6. Ruiz-Alonso M, Blesa D, Díaz-Gimeno P, Gómez E, FernándezSánchez M, Carranza F, et al. The endometrial receptivity array for diagnosis and personalized embryo transfer as a treatment for patients with repeated implantation failure. Fertil Steril. 2013;100(3): 818-24.

7. Hashimoto T, Koizumi M, Doshida M, Toya M, Sagara E, Oka N, et al. Efficacy of the endometrial receptivity array for repeated implantation failure in Japan: A retrospective, two-centers study. Reprod Med Biol. 2017;16(3):290-6.

8. Kort JD, McCoy RC, Demko Z, Lathi RB. Are blastocyst aneuploidy rates different between fertile and infertile populations? J Assist Reprod Genet. 2018;35(3):403-8.

9. Rubio C, Rodrigo L, Garcia-Pascual C, Peinado V, CamposGalindo I, Garcia-Herrero S, Simón C Clinical application of embryo aneuploidy testing by NGS. Biol Reprod. 2019.

10. Franasiak JM, Forman EJ, Hong KH, Werner MD, Upham KM, Treff NR, et al. The nature of aneuploidy with increasing age of the female partner: A review of 15,169 consecutive trophectoderm biopsies evaluated with comprehensive chromosomal screening. Fertil Steril. 2014;101:656-663.e1. 
11. Shapiro BS, Daneshmand ST, Desai J, Garner FC, Aguirre M, Hudson C. The risk of embryo-endometrium asynchrony increases with maternal age after ovarian stimulation and IVF. Reprod Biomed Online. 2016;33(1):50-5.

12. Coughlan C, Ledger W, Wang Q, Liu F, Demirol A, Gurgan T, et al. Recurrent implantation failure: definition and management. Reprod Biomed Online. 2014;28(1):14-38.

13. Martínez F, Latre L, Clua E, Rodriguez I, Coroleu B. Replacing GnRH agonists with GnRH antagonists in oocyte recipient cycle did not adversely affect the pregnancy rates. Eur J Obstet Gynecol Reprod Biol. 2011;159:355-8.

14. Clua E, Tur R, Coroleu B, Boada M, Barri PN, Veiga A. Analysis of factors associated with multiple pregnancy in an oocyte donation programme. Reprod Biomed Online. 2010;21(5):694-9.

15. Martínez F, Kava-Braverman A, Clúa E, Rodríguez I, Gaggiotti Marre S, Coroleu B, et al. Reproductive outcomes in recipients are not associated with oocyte donor body mass index up to 28 $\mathrm{kg} / \mathrm{m} 2$ : a cohort study of 2722 cycles. Reprod Biomed Online. 2017;35(6):739-46.

16. Simon AL, Kiehl M, Fischer E, Proctor JG, Bush MR, Givens C, et al. Pregnancy outcomes from more than 1,800 in vitro fertilization cycles with the use of 24-chromosome single-nucleotide polymorphism-based preimplantation genetic testing for aneuploidy. Fertil Steril. 2018;110(1):113-21.

17. Polanski LT, Baumgarten MN, Quenby S, Brosens J, Campbell BK, Raine-Fenning NJ. What exactly do we mean by "recurrent implantation failure"? A systematic review and opinion. Reprod Biomed Online. 2014;28(4):409-23.

18. Martinez F, Rodriguez I, Devesa M, Buxaderas R, Gómez MJ, Coroleu B. Should progesterone on the human chorionic gonadotropin day still be measured? Fertil Steril. 2015;105:86-92.

19. Coll L, Parriego M, Boada M, Devesa M, Arroyo G, Rodríguez I, et al. Transition from blastomere to trophectoderm biopsy: Comparing two preimplantation genetic testing for aneuploidies strategies. Zygote. 2018;26:191-8.

20. Permanente del Grupo de Interés de Embriología C, Hurtado de Mendoza M, Arroyo G, Figueroa M, Busquets A, De los Santos
M, et al. Cuadernos de embriología clínica II. Criterios ASEBIR de valoración morfológica de oocitos, embriones tempranos y blastocistos humanos. 3rd ed. Madrid: Góbalo; 2015.

21. Cobo A, De Los Santos MJ, Castellò D, Gámiz P, Campos P, Remohí J. Outcomes of vitrified early cleavage-stage and blastocyst-stage embryos in a cryopreservation program: Evaluation of 3,150 warming cycles. Fertil Steril. 2012;98(5): 1138-1146.e1.

22. Coroleu B, Carreras O, Veiga A, Martell A, Martinez F, Belil I, et al. Embryo transfer under ultrasound guidance improves pregnancy rates after in-vitro fertilization. Hum Reprod. 2000;15:616-20.

23. Frantz S, Parinaud J, Kret M, Rocher-Escriva G, PapaxanthosRoche A, Creux H, et al. Decrease in pregnancy rate after endometrial scratch in women undergoing a first or second in vitro fertilization. A multicenter randomized controlled trial. Hum Reprod. 2019;34(1):92-9.

24. Tan J, Kan A, Hitkari J, Taylor B, Tallon N, Warraich G, et al. The role of the endometrial receptivity array (ERA) in patients who have failed euploid embryo transfers. J Assist Reprod Genet. 2018;35(4): 683-92.

25. Reignier A, Lammers J, Barriere P, Freour T. Can time-lapse parameters predict embryo ploidy? A systematic review. Reprod Biomed Online. 2018;36(4):380-7.

26. Zhang J, Tao W, Liu H, Yu G, Li M, Ma S, et al. Morphokinetic parameters from a time-lapse monitoring system cannot accurately predict the ploidy of embryos. J Assist Reprod Genet. 2017;34(9): 1173-8.

27. Nastri C, Lensen S, Gibreel A, Raine-Fenning N, Ferriani R, Bhattacharya $\mathrm{S}$, et al. Endometrial injury in women undergoing assisted reproductive techniques. Cochrane Database Syst Rev. 2015;(3):CD009517.

Publisher's note Springer Nature remains neutral with regard to jurisdictional claims in published maps and institutional affiliations. 